\title{
MicroRNA profiling of platelets from immune thrombocytopenia and target gene prediction
}

\author{
GANG DENG ${ }^{1,2^{*}}$, SHIFANG YU ${ }^{3 *}$, YUNLEI HE ${ }^{2}$, TAO SUN ${ }^{3}$, WEI LIANG ${ }^{2}$, LU YU $^{2}$, DEYI XU ${ }^{2}$, \\ QIANG $\mathrm{LI}^{4}$ and RI ZHANG ${ }^{1}$ \\ ${ }^{1}$ Department of Hematology, The First Affiliated Hospital of Soochow University, Soochow, Jiangsu 215006; \\ ${ }^{2}$ The Ningbo Central Blood Station, Ningbo, Zhejiang 315010; ${ }^{3}$ Department of Transfusion Medicine, \\ The Second Affiliated Hospital of Zhejiang University School of Medicine, Hangzhou, \\ Zhejiang $310009 ;{ }^{4}$ Department of Laboratory Medicine, The First Affiliated Hospital of \\ Zhejiang Chinese Medical University, Hangzhou, Zhejiang 310006, P.R. China
}

Received June 22, 2016; Accepted April 24, 2017

DOI: $10.3892 / \mathrm{mmr} .2017 .6901$

\begin{abstract}
Immune thrombocytopenia (ITP) is an autoimmune disease characterized by a low platelet count and insufficient platelet production. Previous studies identified that microRNAs (miRNAs/miRs) are important for platelet function. However, the regulatory role of miRNAs in the pathogenesis of thrombocytopenia in ITP remains unclear. The aim of the present study is to isolate differentially expressed miRNAs, and identify their roles in platelets from ITP. A total of $5 \mathrm{ml}$ blood from 22 patients with ITP and 8 healthy controls was isolated for platelet collection. A microarray assay was performed to analyze the differentially expressed miRNAs in the patients with ITP and healthy patients. Furthermore, the expression of differentially expressed miRNAs was verified by reverse transcription-quantitative polymerase chain reaction. In addition, the target mRNAs of the differentially expressed miRNAs were predicted via miRWalk databases, and the target genes and miRNAs were classified by Gene Ontology enrichment and Kyoto Encyclopedia of Genes and Genomes analyses. In the present study, 115 miRNAs were identified to be differentially expressed in platelets from patients with ITP compared with the healthy controls $(>3$-fold alteration; $\mathrm{P}<0.05)$. Among
\end{abstract}

Correspondence to: Professor $\mathrm{Ri}$ Zhang, Department of Hematology, The First Affiliated Hospital of Soochow University, 188 Shizi Street, Soochow, Jiangsu 215006, P.R. China

E-mail: dgflying@126.com

Professor Qiang Li, Department of Laboratory Medicine, The First Affiliated Hospital of Zhejiang Chinese Medical University, 54 Youdian Road, Hangzhou, Zhejiang 310006, P.R. China

E-mail: wswzyjs@126.com

*Contributed equally

Key words: microRNA, platelet, microarray, reverse transcription-quantitative polymerase chain reaction, immune thrombocytopenia them, 57 miRNAs were upregulated in ITP, while 58 miRNAs were downregulated. Bioinformatic prediction demonstrated that hsa-miR-548a-5p, hsa-miR-1185-2-3p, hsa-miR-30a-3p, hsa-miR-6867-5p, hsa-miR-765 and hsa-miR-3125 were associated with platelet apoptosis and adhesion in ITP. The present study performed miRNA profiling of platelets from patients with ITP and the results may aid in the understanding of the regulatory mechanism of ITP.

\section{Introduction}

Immune thrombocytopenia (ITP) is currently defined as an autoimmune disease, characterized by a decreased platelet count (due to autoantibodies mediating platelet destruction and insufficient platelet production), which results in purpura and increase of bleeding tendency (1). However, the pathogenesis of ITP remains to be completely elucidated. A previous study demonstrated that ITP was not always associated with a decline in platelet number (2).

Platelets are essential for proper hemostasis and thrombosis. Although platelets lack nucleus, they contain all other necessary components to perform transcription and translation in a signal-dependent manner (3-6). Furthermore, researchers identified that platelets contain abundant and diverse microRNAs (miRNAs/miRs), the key regulators in gene expression alterations (7). Extensive studies were performed to understand the transcriptome of platelets using microarrays or an RNA deep sequencing approach $(8,9)$. miRNAs are present in platelets in variable quantities, and are diverse in humans with specific phenotypes and in different disease states $(10,11)$. Among them, hsa-miR-96 regulated the expression of vesicle-associated membrane protein 8 (also known as endobrevin) (12). However, miRNA targets in ITP are unknown.

Increasing evidence has demonstrated that the expression of aberrant miRNAs is associated with the pathogenesis of ITP $(13,14)$. However, the association between miRNAs and the decrease in platelets in patients with ITP was poorly investigated. In the present study differentially expressed miRNAs were investigated in platelets from patients with ITP and 
healthy control patients. Furthermore, the regulatory network of miRNA-targets was established based on the information from the differentially expressed miRNAs (hsa-miR-548a-5p, hsa-miR-1185-2-3p, hsa-miR-30a-3p, hsa-miR-6867-5p, hsa-miR-765 and hsa-miR-3125) identified. The present analyses may be important in the understanding of the mechanisms of ITP, as well as future therapy.

\section{Materials and methods}

Ethics statement. The present study was approved by the Ethics Committee of Soochow University (Soochow, China) and written informed consent was obtained from all the patients and healthy donors involved.

Subjects. A total of 22 patients with ITP, and 8 age- and sexual-matched healthy donators were recruited from the First Affiliated Hospital of Soochow University (between March 1 and December 31 2015; Table I). The diagnosis of ITP was based on the criteria of the American Society of Hematology (15) and thrombocytopenia was defined as a platelet count of $<50 \times 10^{9}$ platelets/l. The patients with ITP had not received glucocorticoids or immunosuppressive treatment. Patients with the following complications were excluded: Diabetes, hypertension, cardiovascular diseases, pregnancy, active infection or autoimmune diseases other than ITP. Of the 22 ITP samples, 8 samples were studied by microarray together with 8 healthy samples, the other 14 ITP patient samples were tested using the reverse transcription-quantitative polymerase chain reaction (RT-qPCR) following performance of the microarray. Prior to the microarray, the platelet concentrations from 8 patients with ITP were adjusted to $100 \times 10^{9}$ platelets $/ 1$. A total of $1 \mathrm{ml}$ each sample was used for the ITP groups, for a total of $8 \mathrm{ml}$. The platelets for the control groups were processed in the same way, for a total of $8 \mathrm{ml}$.

Preparation of leukocyte-depleted apheresis platelets (LDPS). LDPs were prepared as previously reported (14). To deplete white blood cells (WBCs), reticulocytes and red blood cells (RBCs), the platelets were treated with pan-leukocyte [anti-cluster of differentiation (CD) $45^{+}$, anti-CD71 ${ }^{+}$, and anti-CD235'] immunomagnetic beads, according to the manufacturers instruction (Invitrogen, Carlsbad, CA, USA). Following treatment, WBCs, RBCs and reticulocytes were not detected by flow cytometry (16). The leukocyte-depleted platelets from 8 ITP patients and 8 health controls were pooled, respectively.

RNA extraction. Total RNA was extracted using an miRNA isolation kit (Beijing CoWin Biotech Co., Ltd., Beijing, China), according to the manufacturer's protocol. The quantity and purity was determined using a NanoDrop 2000 spectrophotometer (Thermo Fisher Scientific, Inc., Waltham, MA, USA) and Agilent Bioanalyzer 2100 (Agilent Technologies, Inc., Santa Clara, CA, USA), respectively.

miRNA microarray analysis. The Agilent Human miRNA (8x60K) Array (version 21.0; design ID: 70156; Agilent Technologies, Inc.), which covers 2549 human miRNAs (based on miRBase release 21.0) (17), was used to detect
miRNA expression in platelets. Microarray experiments were performed by Shanghai Biotechnology Company (www. ebioservice.com; Shanghai, China). Normalization was performed using Gene Spring software (version 11.0; Agilent Technologies, Inc.). Student's t-tests were used in the gene screening. $\mathrm{P}<0.05$ was considered to indicate a statistically significant difference, and the fold change threshold values were $>3.0$ and $<0.33$. Hierarchical clustering was performed to generate miRNA and sample trees based on Pearson correlation using $\mathrm{MeV}$ software (version 4.0; Multi Experiment Viewer; www.tm4.org/\#/welcome).

miRNA RT-qPCR analysis. A total of 9 differentially expressed miRNAs identified by microarray were selected for further validation using RT-qPCR. For the reverse transcription of total RNA, the miRNA cDNA kit (Beijing CoWin Biotech Co., Ltd.) was used, according to the manufacturer's protocol. Total RNAs were initially treated with Escherichia coli poly-A polymerase to generate a poly-A tail at the 3'-end of each miRNA. Following polyadenylation, the miRNA first strand cDNA was synthesized using the poly (T) adapter (GCGAGCACAGAATTAATACGACTCACT ATAGGTTTTTTTTTTTTVN) as primer, at $42^{\circ} \mathrm{C}$ for $1 \mathrm{~h}$. To measure the expres sion of mature miRNAs, the miRNA-first strand cDNAs was analyzed using the miRNA Real-Time PCR Assay kit (Beijing CoWin Biotech Co., Ltd.) and a StepOnePlus $^{\mathrm{TM}}$ Real-Time PCR system (Applied Biosystems; Thermo Fisher Scientific, Inc.). The primers for the RT-qPCR analysis are listed in Table II. Results were normalized to $5 \mathrm{~S}$ ribosomal RNA. The thermocycling conditions were $95^{\circ} \mathrm{C}$ for $10 \mathrm{~min}$, followed by 40 cycles of $95^{\circ} \mathrm{C}$ for $15 \mathrm{sec}$ and $60^{\circ} \mathrm{C}$ for $1 \mathrm{~min}$. The data were quantified using the $2^{-\Delta \Delta \mathrm{Cq}}$ method (18). The data were processed using StepOne ${ }^{\mathrm{TM}}$ software (version 2.2.2; Applied Biosystems; Thermo Fisher Scientific, Inc.).

Prediction of target genes of differentially expressed miRNAs. The target genes of the candidate miRNAs were predicted using online tools contained within miRWalk software (www.umm.uni-heidelberg.de/apps/zmf/mirwalk) (19) and six bioinformatic algorithms (DIANAmT, miRanda, miRDB, miRWalk, PicTar and TargetScan).

Gene Ontology (GO) and Kyoto Encyclopedia of Genes and Genomes (KEGG) analyses. To further understand the biological function of differentially expressed miRNAs, the Gene Ontology and KEGG analyses were conducted using the Database for Annotation, Visualization and Integrated Discovery online analysis tool (20) and GENECODIS (21). Fisher's exact test and $\chi^{2}$ tests were used to select the significant GO category orKEGG pathway, and the false discovery rate (FDR) was calculated to correct the p-value. FDR $<0.01$ and $\mathrm{P}<0.01$ were considered to be statistically significant.

Regulatory network construction between miRNAs and their targets. The post-transcriptional regulatory network is defined as a directed and bipartite graph in which expressions of miRNAs and their targets are reversely correlated. A regulatory network of miRNAs and their potential targets was presented using Cytoscape software (22). 
Table I. Clinical characteristics of the patients with ITP and healthy controls.

\begin{tabular}{|c|c|c|c|c|c|c|}
\hline Patient no. & Sex & Age, years & Bleeding symptoms & PLT count, PLT $x 10^{9} / 1$ & Group & Test \\
\hline 1 & $\mathrm{~F}$ & 78 & $\mathrm{EC}, \mathrm{GH}$ & 33 & ITP & Microarray \\
\hline 2 & M & 45 & $\mathrm{EC}, \mathrm{EP}$ & 42 & ITP & Microarray \\
\hline 3 & M & 56 & PT & 40 & ITP & Microarray \\
\hline 4 & $\mathrm{~F}$ & 37 & PT, EC & 35 & ITP & Microarray \\
\hline 5 & M & 53 & PT & 43 & ITP & Microarray \\
\hline 6 & $\mathrm{~F}$ & 52 & $\mathrm{EC}$ & 28 & ITP & Microarray \\
\hline 7 & $\mathrm{~F}$ & 21 & PT, GH & 28 & ITP & Microarray \\
\hline 8 & $\mathrm{~F}$ & 63 & $\mathrm{EC}, \mathrm{GH}$ & 34 & ITP & Microarray \\
\hline 9 & $\mathrm{~F}$ & 22 & $\mathrm{EC}, \mathrm{EP}$ & 42 & ITP & RT-qPCR \\
\hline 10 & $\mathrm{~F}$ & 33 & PT & 37 & ITP & RT-qPCR \\
\hline 11 & M & 36 & PT, EC & 29 & ITP & RT-qPCR \\
\hline 12 & $\mathrm{~F}$ & 31 & PT & 43 & ITP & RT-qPCR \\
\hline 13 & $\mathrm{~F}$ & 40 & $\mathrm{EC}$ & 34 & ITP & RT-qPCR \\
\hline 14 & $\mathrm{~F}$ & 34 & PT, GH & 36 & ITP & RT-qPCR \\
\hline 15 & M & 38 & PT & 38 & ITP & RT-qPCR \\
\hline 16 & M & 29 & $\mathrm{EC}$ & 45 & ITP & RT-qPCR \\
\hline 17 & $\mathrm{~F}$ & 36 & $\mathrm{EC}, \mathrm{GH}$ & 25 & ITP & RT-qPCR \\
\hline 18 & $\mathrm{~F}$ & 31 & $\mathrm{EC}, \mathrm{GH}$ & 47 & ITP & RT-qPCR \\
\hline 19 & $\mathrm{~F}$ & 40 & $\mathrm{EP}$ & 41 & ITP & RT-qPCR \\
\hline 20 & M & 63 & PT & 38 & ITP & RT-qPCR \\
\hline 21 & $\mathrm{~F}$ & 22 & PT & 42 & ITP & RT-qPCR \\
\hline 22 & M & 33 & $\mathrm{EC}, \mathrm{GH}$ & 41 & ITP & RT-qPCR \\
\hline 23 & $\mathrm{~F}$ & 36 & NA & 157 & Healthy control & Microarray, RT-qPCR \\
\hline 24 & $\mathrm{~F}$ & 31 & NA & 252 & Healthy control & Microarray, RT-qPCR \\
\hline 25 & $\mathrm{~F}$ & 40 & NA & 383 & Healthy control & Microarray, RT-qPCR \\
\hline 26 & M & 34 & NA & 292 & Healthy control & Microarray, RT-qPCR \\
\hline 27 & $\mathrm{~F}$ & 38 & NA & 371 & Healthy control & Microarray, RT-qPCR \\
\hline 28 & M & 29 & NA & 198 & Healthy control & Microarray, RT-qPCR \\
\hline 29 & $\mathrm{~F}$ & 36 & NA & 229 & Healthy control & Microarray, RT-qPCR \\
\hline 30 & $\mathrm{~F}$ & 31 & NA & 257 & Healthy control & Microarray, RT-qPCR \\
\hline
\end{tabular}

ITP, immune thrombocytopenia; F, female; M, male; PT, petechiae; EC, ecchymoses; EP, epistaxis; GH, genitourinary hemorrhage; NA, not applicable; PLT, platelet; RT-qPCR, reverse transcription-quantitative polymerase chain reaction.

\section{Results}

Identification of the differentially expressed miRNAs in patients with ITP. ITP is a severe disease that affects humans, previous results have demonstrated that miRNAs may serve an important role in ITP pathogenesis (23-25). However, the role of platelet-derived miRNAs in ITP remains to be examined. To determine miRNA alterations in ITP platelets compared with the healthy controls, microarray analysis was performed. The results demonstrated that 537 and 544 miRNAs were expressed in the ITP and control samples, respectively. Among them, 115 miRNAs were differentially expressed (fold-change of $>3$ or $<0.33 ; \mathrm{P}<0.05)$. Of these, 57 miRNAs were upregulated while 58 miRNAs were downregulated in the ITP samples compared with the healthy samples.

Of the differentially expressed miRNAs, hsa-miR-338-5p, hsa-miR-765, hsa-miR-122-5p and hsa-miR-451b (specifically expressed in ITP), and hsa-miR-133a-3p,
hsa-miR-224-3p, hsa-miR-452-5p, hsa-miR-491-5p and hsa-miR-15a-3p (restricted to control group) were verified by RT-qPCR analysis. The RT-qPCR results demonstrated similar patterns as observed in the microarray data (Fig. 1). The top 20 differentially expressed miRNAs are listed in Table III.

Identification of the targets of differentially expressedmiRNAs. To identify the target genes of the differentially expressed miRNAs in ITP, bioinformatic prediction was performed using the miRWalk database. A total of 677 pairs of miRNA-target genes were identified for the upregulated miRNAs and 1,274 pairs for the downregulated miRNAs (data not shown).

GO and KEGG analyses of target genes of downregulated miRNAs in ITP. GO functional and KEGG pathway analyses were performed for the 1,274 target genes of the downregulated ITP miRNAs. GO terms were assigned to the potential targets. The GO terms associated with the targets were categorized 
A

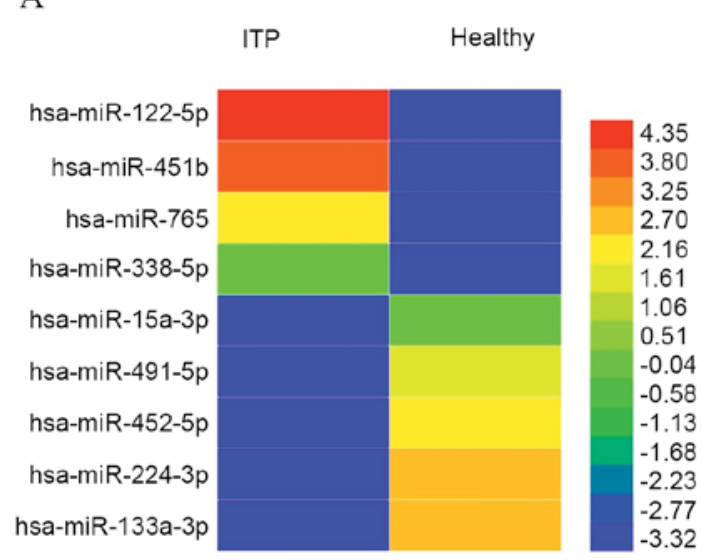

B

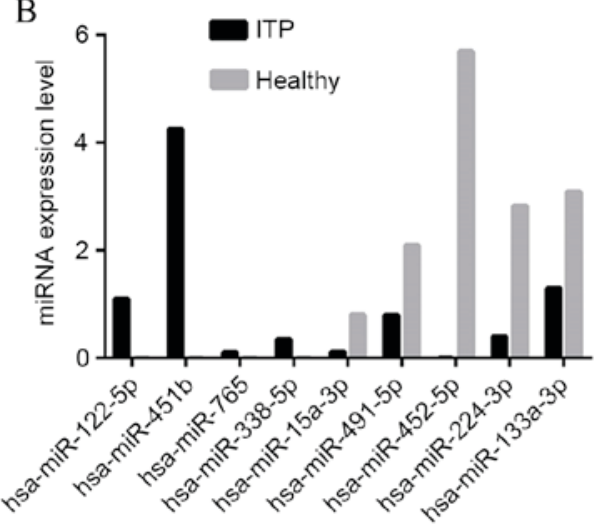

$\mathrm{C}$

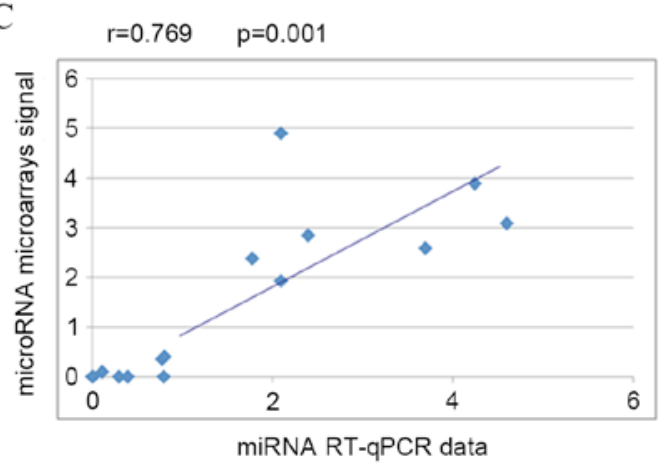

Figure 1. A total of 9 differentially expressed miRNAs were further verified by RT-qPCR. (A) Heatmap of the differentially expressed miRNAs' microarray data. (B) The RT-qPCR data of the differentially expressed miRNAs. (C) The data demonstrated that there is a correlation between the RT-qPCR data and the microarray signal $(\mathrm{r}=0.769 ; \mathrm{P}=0.001)$. $\mathrm{miRNA} / \mathrm{miR}$, microRNA; ITP, immune thrombocytopenia.

Table II. List of primers used for the reverse transcriptionquantitative polymerase chain reaction analysis.

\section{Gene}

Primer (5' to $3^{\prime}$ )

5S rRNA forward

5S rRNA reverse hsa-miR-338-5p hsa-miR-122-5p hsa-miR-451b hsa-miR-452-5p hsa-miR-15a-3p hsa-miR-548a-5p hsa-miR-30a-3p hsa-miR-765 hsa-miR-765 hsa-miR-224-3p hsa-miR-133a-3p hsa-miR-491-5p hsa-miR-3125 hsa-miR-1185-2-3p hsa-miR-6867-5p Universal reverse primer
$(\mathrm{FDR}<0.01 ; \mathrm{P}<0.01)$ into 16 classes. In order to better understand the function of the involved genes, these GO terms were divided into three groups including biological processes (BP; 10 classes), molecular function (MF; 2 classes) and cellular components ( $\mathrm{CC} ; 4$ classes). In the BP group, the major regulatory pathways included GO:0010604-positive regulation of macromolecular metabolic processes, GO:0009891-positive regulation of biosynthetic processes, GO:0042127-regulation of cellular proliferation, GO:0031328-positive regulation of cellular biosynthetic processes, GO:0010557-positive regulation of macromolecular biosynthetic processes, GO:0043067-regulation of programmed cell death, GO:0010941-regulation of cell death, GO:0051173-positive regulation of nitrogen compound metabolic processes, GO:0042981-regulation of apoptosis and GO:0010628-positive regulation of gene expression (Fig. 2A). The most predicted results were involved with apoptosis and cell death.

To better understand the function of potential targets, signaling pathways were analyzed using the KEGG database (24 signaling pathways). The pathways associated with the downregulated miRNAs in ITP $(\mathrm{P}<0.01)$ included the Wnt signaling pathway (KEGG pathway, hsa04,310; $\mathrm{P}=2.24 \times 10^{-5}$ ), the global cancer pathway map (KEGG pathway, hsa05,200; $\left.\mathrm{P}=1.93 \times 10^{-4}\right)$, a small cell lung cancer-associated pathway (KEGG pathway, hsa05, 222; $\mathrm{P}=1.20 \times 10^{-3}$ ), the mechanistic target of rapamycin signaling pathway (KEGG pathway, hsa04, 150; $\left.\mathrm{P}=3.11 \times 10^{-3}\right)$, a pancreatic cancer-associated pathway (KEGG pathway, hsa05212; $\mathrm{P}=3.14 \times 10^{-3}$ ).

rRNA, ribosomal RNA; miR, microRNA. 
Table III. Top 20 differentially expressed miRs.

\begin{tabular}{lc}
\hline A, Upregulated & \\
\hline miR & Fold change \\
\hline hsa-miR-4730 & 305.53 \\
hsa-miR-122-5p & 297.44 \\
hsa-miR-6716-3p & 273.18 \\
hsa-miR-575 & 230.61 \\
hsa-miR-6867-5p & 219.03 \\
hsa-miR-4800-5p & 208.65 \\
hsa-miR-4778-5p & 180.35 \\
hsa-miR-4716-3p & 179.61 \\
hsa-miR-5581-5p & 164.55 \\
hsa-miR-6767-5p & 159.14 \\
hsa-miR-451b & 147.17 \\
hsa-miR-4653-3p & 131.93 \\
hsa-miR-5088-5p & 120.57 \\
hsa-miR-6751-3p & 115.94 \\
hsa-miR-6512-5p & 105.84 \\
hsa-miR-6873-3p & 104.97 \\
hsa-miR-5194 & 104.28 \\
hsa-miR-4634 & 96.54 \\
hsa-miR-3125 & 94.56 \\
hsa-miR-3607-3p & 92.27 \\
\hline
\end{tabular}

B, Downregulated

$\operatorname{miR}$ Fold change

\begin{tabular}{ll}
\hline hsa-miR-299-3p & 0.01 \\
hsa-miR-487a-3p & 0.01 \\
hsa-miR-370-3p & 0.01 \\
hsa-miR-136-3p & 0.01 \\
hsa-miR-1307-5p & 0.01 \\
hsa-miR-133a-3p & 0.01 \\
hsa-miR-1185-2-3p & 0.01 \\
hsa-miR-3174 & 0.01 \\
hsa-miR-412-5p & 0.01 \\
hsa-miR-3194-5p & 0.01 \\
hsa-miR-224-3p & 0.01 \\
hsa-miR-548a-5p & 0.01 \\
hsa-miR-30a-3p & 0.02 \\
hsa-miR-3617-5p & 0.02 \\
hsa-miR-542-3p & 0.02 \\
hsa-miR-9-3p & 0.02 \\
hsa-miR-452-5p & 0.02 \\
hsa-miR-5187-5p & 0.02 \\
hsa-miR-766-5p & 0.02 \\
hsa-miR-100-5p & 0.02 \\
\hline
\end{tabular}

miR, microRNA.

GO and KEGG analyses of target genes of upregulated miRNAs in ITP. GO enrichment and KEGG pathway analyses were performed for the 677 target genes of the upregulated ITP miRNAs. The GO terms associated with the targets were categorized into 5 classes $(\mathrm{FDR}<0.20$; $\mathrm{P}<0.01$ ), including BP (4 classes; GO:0007156-homophilic cell adhesion, GO:0016337-cell-cell adhesion, GO:0007155-cell adhesion, GO:0022610-biological adhesion) and CC (1 class; CC-GO:0005886-plasma membrane) (Fig. 2B).

To better understand the function of potential targets, signaling pathways were analyzed using the KEGG database (7 signaling pathways; Fig. 3). The pathway associated with the upregulated miRNAs in ITP $(\mathrm{P}<0.05)$ was the cell adhesion molecules (CAMs) pathway, it was significantly enriched in the analysis (hsa04, 514; $\mathrm{P}=1.47 \times 10^{-2}$ ).

Regulatory network of miRNAs and their target genes. In order to investigate the association among the miRNAs of interest, the miRNA target gene regulatory network in ITP was created using Cytoscape software. The upregulated miRNAs, downregulated miRNAs and their targets formed a regulatory network. Among the downregulated miRNAs, hsa-miR-548a-5p exhibited the maximum number of target genes (50 genes; Fig. 3). Among the upregulated miRNAs, hsa-miR-6867-5p possessed 24 regulatory target genes, while hsa-miR-765 and hsa-miR-3125 targeted 18 and 9 genes, respectively (Fig. 4).

\section{Discussion}

The functions of platelets, including activation, adhesion and aggregation, are crucial for coagulation physiology and the maintenance of hemostatic balance. Platelet dysfunction is associated with various pathologies, including atherosclerosis, occlusive or thrombotic hemorrhagic disorders (26). The roles of miRNAs in platelets have been given increasing attention due to their importance in ITP pathogenesis. To date, the studies have investigated the roles of miRNAs in biological processes in platelets. Girardot et al (27) demonstrated that hsa-miR-28, as well as other miRNAs, targets receptor of thrombopoietin (MPL) and MPL repression is potentially involved in the regulation of platelet count. Nagalla et al (28) reported that hsa-miR-107 targets clock circadian regulator, which may regulate circadian platelet reactivity. The authors previously demonstrated that hsa-miR-326 served a crucial role in platelet apoptosis during storage (29).

It is well-known that platelets have mRNA and mRNA splicing machinery, and are able to translate mRNA into proteins $(3,30)$. Platelets also contain miRNA processing machinery, including endoribonuclease dicer (DICER1), TAR RNA-binding protein 2 and protein argonaute-2, which is involved in the processing of pre-miRNA into mature miRNA (7). Microarray-based studies demonstrated that $\leq 32 \%$ of human mRNAs were expressed in platelets $(31,32)$. Several studies have focused on the analysis of the platelet transcriptome (6,31-34) and deduced a certain correlation between mRNA and coupled proteins $(31,34)$. The mRNA in platelets originated from megakaryocytes and seem to be affected by aging and platelet activation $(8,35)$. Zhang et al (36) observed that 6 marker proteins with significant differences in the platelet lysates of patients with primary ITP patients compared with the secondary ITP and healthy controls. 
A

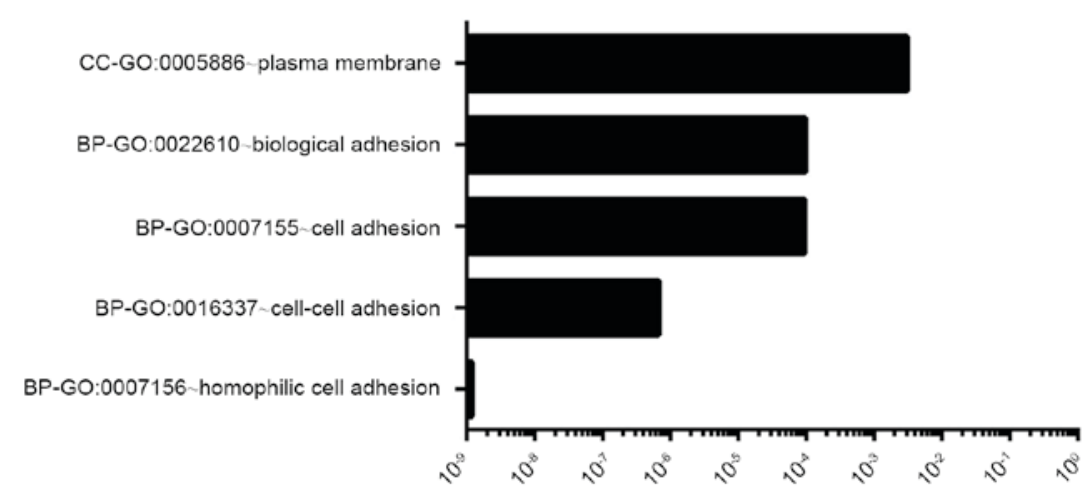

B

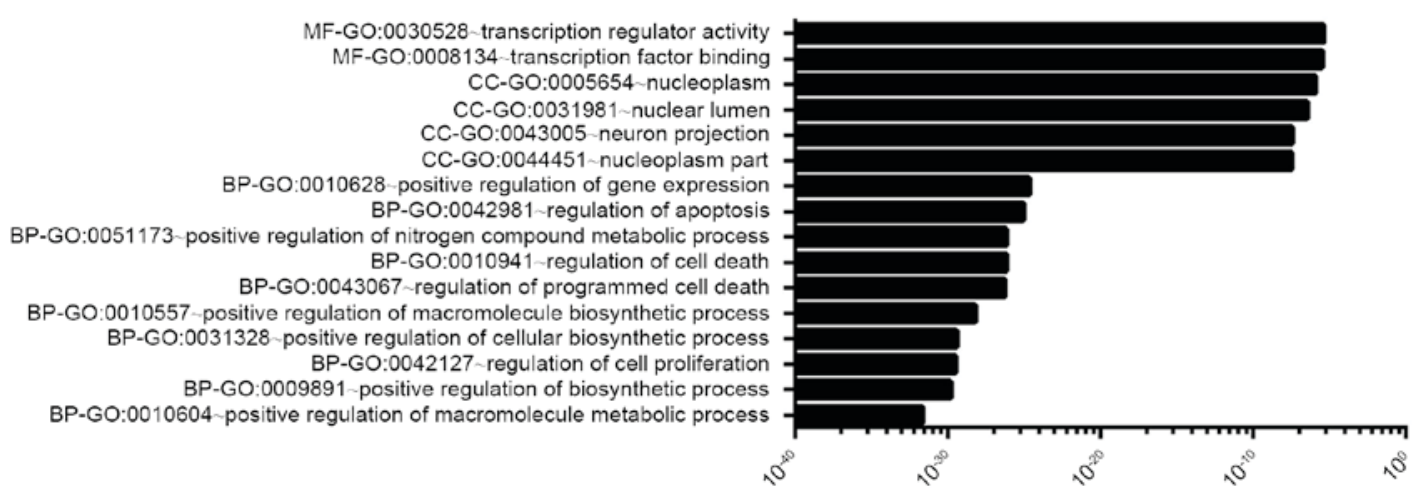

Figure 2. GO BP were investigated by mapping the predicted target genes. $\mathrm{P}<0.01$ was used as a threshold to select significant GO terms and Kyoto Encyclopedia of Genes and Genomes pathways. (A) GO terms of upregulated miRNAs according to - $\log$ (P-value) were annotated. (B) GO terms of downregulated miRNAs according to - $\log (\mathrm{P}$-value) were annotated. GO, gene ontology; BP, biological processes; CC, cellular components; MF, molecular function; miRNA, microRNA.

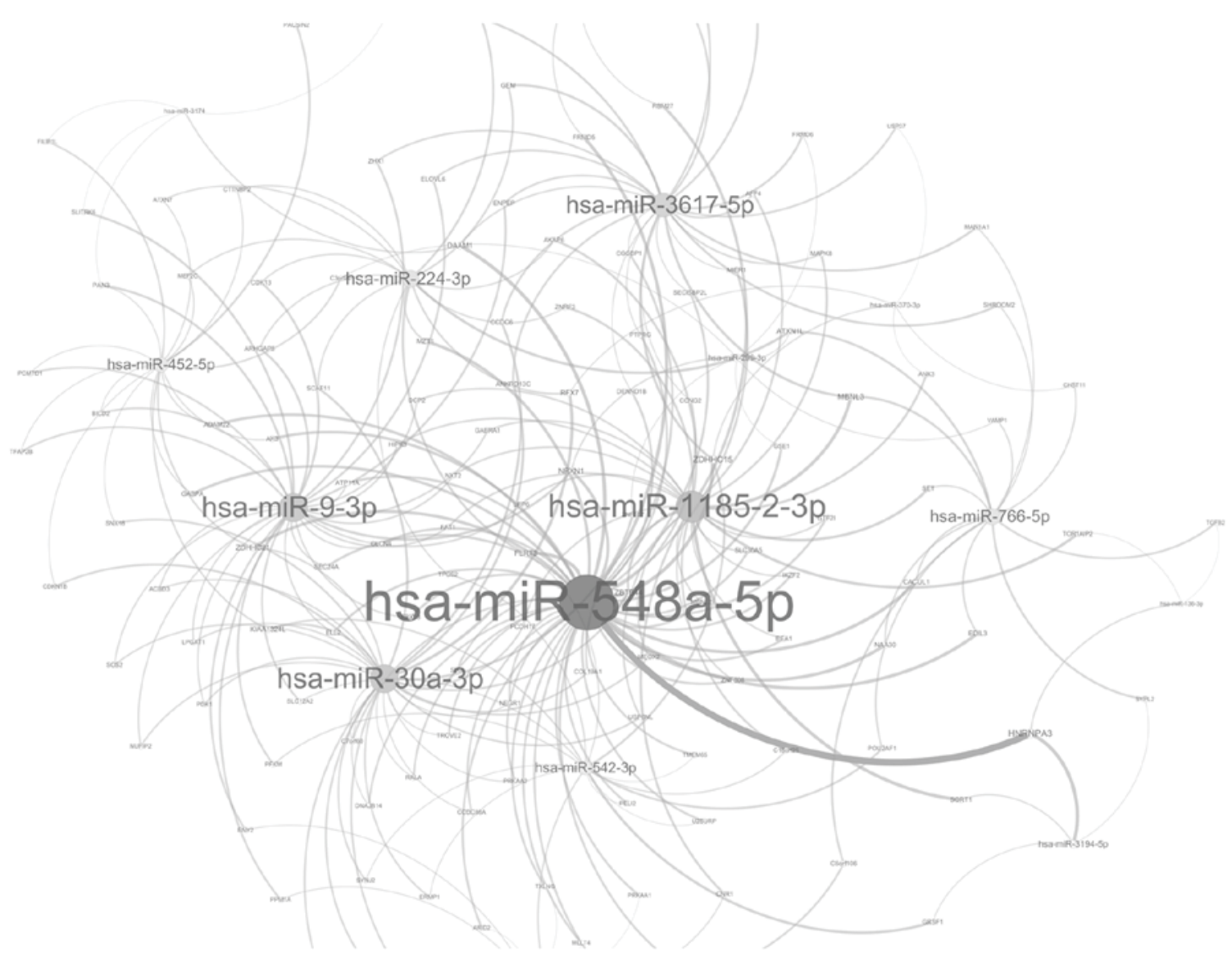

Figure 3. Regulatory networks between downregulated miRs and their target genes in platelets from patients with immune thrombocytopenia. miR, microRNA. 
Table IV. Comparison between the top 20 differentially expressed platelet miRNAs in the present study and the report by Osman and Falker (36).

Characterization of human platelet miRNA by reverse transcription-quantitative polymerase chain ITP (miRbase 21) Healthy control (miRbase 21) reaction analysis (miRbase 14)

\begin{tabular}{lll}
\hline hsa-miR-7975 & hsa-miR-7975 & hsa-miR-223-3p \\
hsa-miR-7977 & hsa-miR-7977 & hsa-miR-92 \\
hsa-miR-5100 & hsa-miR-223-3p & hsa-miR-16-5p \\
hsa-miR-223-3p & hsa-miR-21-5p & hsa-miR-126-3p \\
hsa-miR-1260a & hsa-miR-103a-3p & hsa-miR-142-3p \\
hsa-miR-21-5p & hsa-let-7a-5p & hsa-miR-26a-5p \\
hsa-miR-16-5p & hsa-miR-16-5p & hsa-miR-565 \\
hsa-miR-451a & hsa-let-7f-5p & hsa-miR-484 \\
hsa-miR-6090 & hsa-miR-26a-5p & hsa-miR-486 \\
hsa-let-7a-5p & hsa-let-7b-5p & hsa-miR-222 \\
hsa-miR-103a-3p & hsa-miR-107 & hsa-miR-451a \\
hsa-let-7f-5p & hsa-miR-5100 & hsa-miR-191 \\
hsa-miR-4286 & hsa-miR-24-3p & hsa-miR-24-3p \\
hsa-miR-6089 & hsa-miR-130a-3p & hsa-miR-650 \\
hsa-miR-1273g-3p & hsa-miR-126-3p & hsa-miR-93 \\
hsa-miR-126-3p & hsa-miR-23a-3p & hsa-miR-20a-5p \\
hsa-miR-107 & hsa-let-7d-5p & hsa-miR-19b-3p \\
hsa-let-7b-5p & hsa-miR-19b-3p & hsa-miR-26b \\
hsa-miR-7641 & hsa-miR-20a-5p & hsa-miR-17 \\
hsa-miR-23a-3p & hsa-miR-15b-5p & hsa-miR-106b
\end{tabular}

miR, microRNA.

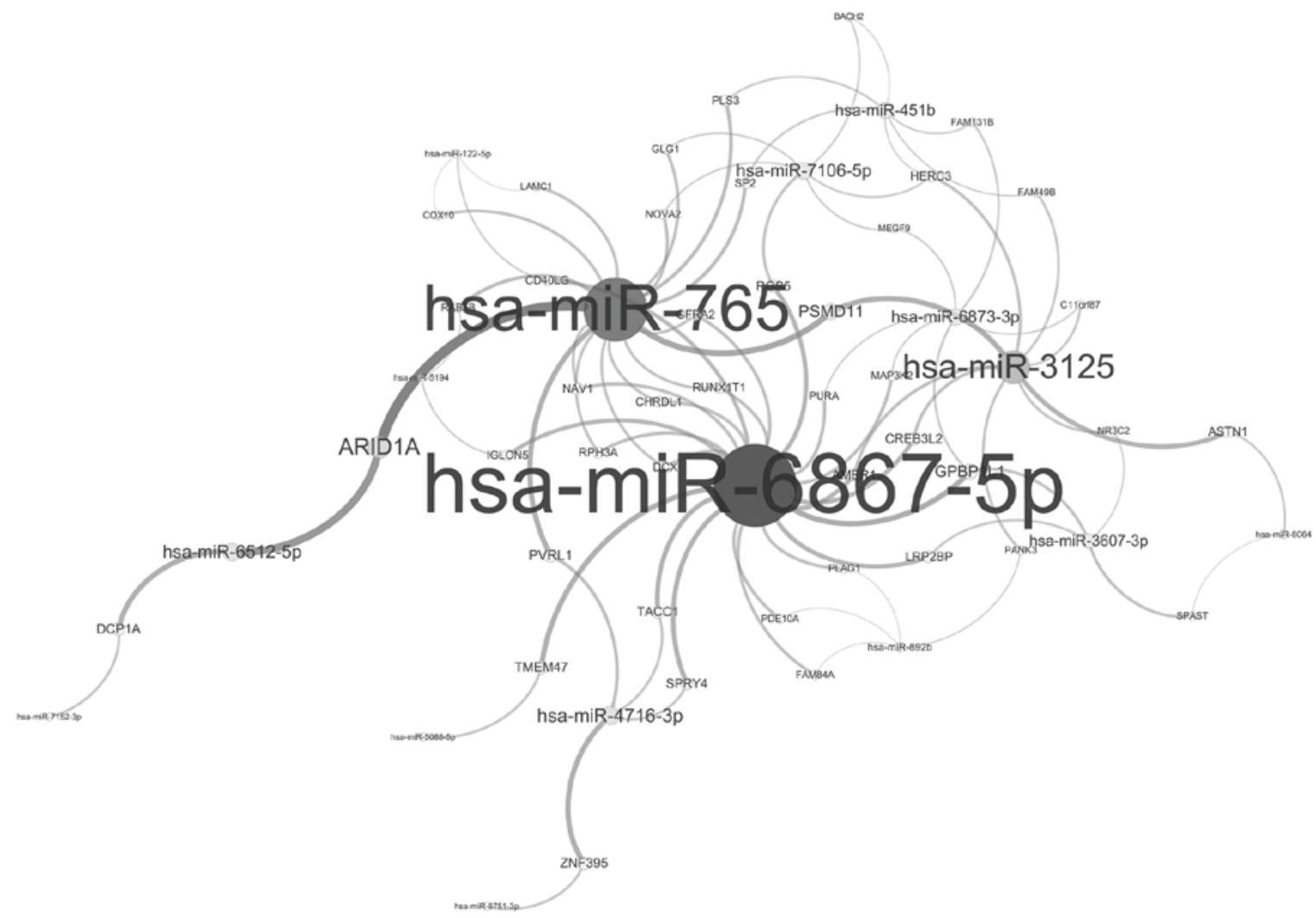

Figure 4. Regulatory networks between upregulated miRs and their target genes in platelets from patients with immune thrombocytopenia. miR, microRNA. 
Patients with ITP exhibit a decreased platelet count accompanied with dysfunction, including increased apoptosis and the reduction of adhesion function (37-39). However, the underlying mechanism of ITP pathogenesis remains unclear. In the present study, the expression of platelet miRNAs was analyzed by microarray. The miRNAs expressed in the platelets of patients with ITP and healthy controls were compared, and there were 115 differentially expressed miRNAs between the two groups. To confirm the reliability of the microarray results, 9 differentially expressed miRNAs were further verified using RT-qPCR. The results of the RT-qPCR data were consistent with the microarray data obtained (Fig. 1). Among a total of 115 differentially expressed miRNAs, 57 miRNAs were upregulated while 58 miRNAs were downregulated in ITP. The observations also suggested that human platelets contain different types of miRNAs, and these were stable and reproducible (Table IV). The data was consisted with the report by Osman and Falker (40).

To better understand the function of miRNAs, bioinformatic analysis was performed, including GO and KEGG pathway analysis. The results revealed that $21 \mathrm{GO}$ terms and 6 signaling pathways were associated with the potential targets $(\mathrm{P}<0.01)$. Networks of $16 \mathrm{GO}$ terms and 5 pathways of interest were built between downregulated miRNAs and their target genes. The results demonstrated that downregulated miRNAs may be involved in platelet apoptosis and cell death. Among these downregulated miRNAs, hsa-miR-548a-5p was the most important modulator and was able to modulate 50 target genes. The targets of hsa-miR-548a-5p, including DICER1, histone acetyltransferase p300, low-density lipoprotein receptor related protein 1B, ADAM metallopeptidase domain 8 (ADAM8), serine carboxypeptidase 1, topoisomerase (DNA) II $\alpha$ and erb-b2 receptor tyrosine kinase 2, were involved in apoptosis. Zhang et al (41) reported that ADAM8 potentially served a role in the pathogenesis of non-small cell lung cancer by resisting cisplatin-mediated apoptosis. Excluding hsa-miR-548a-5p, the other downregulated miRNAs were also predicted to serve important roles in cellular apoptosis. miR-9-3p serves a role in tumor suppression by targeting tafazzin in hepatocellular carcinoma cells. The results of the present study indicated in GO terms that these downregulated miRNAs in ITP may promote platelet apoptosis.

Networks of five GO terms and one pathway of interest were built between upregulated miRNAs and their target genes. The results demonstrated that upregulated miRNAs may be involved in platelet adhesion. Among these upregulated miRNAs, hsa-miR-6867-5p was the most important modulator and was able to modulate 24 target genes. The targets of hsa-miR-6867-5p, including cyclin D1, CD40 ligand, integrin subunit $\alpha 11$ and PLAG1 zinc finger, were involved in cellular adhesion.

Following GO analysis, the KEGG database was employed to analyze the pathways involved in the predicted miRNA target genes. KEGG analysis demonstrated that these signaling pathways were associated with the CAMs pathway. In the present study, the CAMs pathway was the most associated pathway. hsa-miR-6867-5p, hsa-miR-122-5p and hsa-miR-892b may comodulate the CAMs pathway. The results suggested that several miRNAs coparticipate in the same pathways and serve important roles in the cell adhesion of platelets. Previous studies demonstrated that the CAMs pathway was implicated in the pathogenesis of ITP $(42,43)$. The present research implied that miRNAs may serve an important role in the platelet apoptosis of ITP. Further studies are required to provide more information in understanding the underlying mechanism of ITP pathogenesis.

In conclusion, 115 differentially expressed miRNAs in the platelets from patients with ITP and healthy controls were identified. The predication of these differentially expressed miRNAs and their target genes provided information on the understanding of ITP pathogenesis. A number of the miRNA-regulated molecular networks and biological processes identified in the present study are associated with platelet apoptosis and adhesion. The molecular pathways presented in the present study constituted a comprehensive resource for future investigations into the role of specific miRNAs in ITP pathogenesis.

\section{Acknowledgements}

The present study was supported by grants from the Project of Ningbo Medical Science and Technology Plans (grant no. 2016A17), the Ningbo City Natural Science Foundation (grant no. 2,015A610308) and Zhejiang Provincial Natural Science Foundation (grant no. LY16H200005).

\section{References}

1. Neunert CE: Current management of immune thrombocytopenia. Hematology Am Soc Hematol Educ Program 2013: 276-282, 2013.

2. Varga-Szabo D, Pleines I and Nieswandt B: Cell adhesion mechanisms in platelets. Arterioscler Thromb Vasc Biol 28: 403-412, 2008.

3. Denis MM, Tolley ND, Bunting M, Schwertz H, Jiang H, Lindemann S, Yost CC, Rubner FJ, Albertine KH, Swoboda KJ, et al: Escaping the nuclear confines: Signal-dependent pre-mRNA splicing in anucleate platelets. Cell 122: 379-391, 2005.

4. Dittrich M, Birschmann I, Pfrang J, Herterich S, Smolenski A, Walter U and Dandekar T: Analysis of SAGE data in human platelets: Features of the transcriptome in an anucleate cell. Thromb Haemost 95: 643-651, 2006.

5. Schwertz H, Tolley ND, Foulks JM, Denis MM, Risenmay BW, Buerke M, Tilley RE, Rondina MT, Harris EM, Kraiss LW, et al: Signal-dependent splicing of tissue factor pre-mRNA modulates the thrombogenicity of human platelets. J Exp Med 203: 2433-2440, 2006.

6. Rowley JW, Oler AJ, Tolley ND, Hunter BN, Low EN, Nix DA, Yost CC, Zimmerman GA and Weyrich AS: Genome-wide RNA-seq analysis of human and mouse platelet transcriptomes. Blood 118: e101-e111, 2011.

7. Landry P, Plante I, Ouellet DL, Perron MP, Rousseau G and Provost P: Existence of a microRNA pathway in anucleate platelets. Nat Struct Mol Biol 16: 961-966, 2009.

8. Bray PF, McKenzie SE, Edelstein LC, Nagalla S, Delgrosso K, Ertel A, Kupper J, Jing Y, Londin E, Loher P, et al: The complex transcriptional landscape of the anucleate human platelet. BMC Genomics 14: 1, 2013.

9. Londin ER, Hatzimichael E, Loher P, Edelstein L, Shaw C, Delgrosso K, Fortina P, Bray PF, McKenzie SE and Rigoutsos I: The human platelet: Strong transcriptome correlations among individuals associate weakly with the platelet proteome. Biol Direct 9: 3, 2014.

10. Freedman JE, Larson MG, Tanriverdi K, O'Donnell CJ, Morin K, Hakanson AS, Vasan RS, Johnson AD, Iafrati MD and Benjamin EJ: Relation of platelet and leukocyte inflammatory transcripts to body mass index in the Framingham heart study. Circulation 122: 119-129, 2010. 
11. Lood C, Amisten S, Gullstrand B, Jönsen A, Allhorn M, Truedsson L, Sturfelt G, Erlinge D and Bengtsson AA: Platelet transcriptional profile and protein expression in patients with systemic lupus erythematosus: Up-regulation of the type I interferon system is strongly associated with vascular disease. Blood 116: 1951-1957, 2010.

12. Gatsiou A, Boeckel JN, Randriamboavonjy V and Stellos K: MicroRNAs in platelet biogenesis and function: Implications in vascular homeostasis and inflammation. Curr Vasc Pharmacol 10: 524-531, 2012.

13. Burenbatu, Borjigin M, Eerdunduleng, Huo W, Gong C, Hasengaowa, Zhang G, Longmei, Li M, Zhang X, et al: Profiling of miRNA expression in immune thrombocytopenia patients before and after Qishunbaolier (QSBLE) treatment. Biomed Pharmacother 75: 196-204, 2015.

14. Qian BH, Ye X, Zhang L, Sun Y, Zhang JR, Gu ML, Qin Q, Chen J and Deng AM: Increased miR-155 expression in peripheral blood mononuclear cells of primary immune thrombocytopenia patients was correlated with serum cytokine profiles. Acta Haematol 133: 257-263, 2015.

15. Rodeghiero F, Stasi R, Gernsheimer T, Michel M, Provan D, Arnold DM, Bussel JB, Cines DB, Chong BH, Cooper N, et al: Standardization of terminology, definitions and outcome criteria in immune thrombocytopenic purpura of adults and children Report from an international working group. Blood 113: 2386-2393, 2009.

16. Yu S, Deng G, Qian D, Xie Z, Sun H, Huang D and Li Q: Detection of apoptosis-associated microRNA in human apheresis platelets during storage by quantitative real-time polymerase chain reaction analysis. Blood Transfus 12: 541-547, 2014.

17. Kozomara A and Griffiths-Jones S: miRBase: Annotating high confidence microRNAs using deep sequencing data. Nucleic Acids Res 42 (Database issue): D68-D73, 2014.

18. Livak KJ and Schmittgen TD: Analysis of relative gene expression data using real-time quantitative PCR and the 2(-Delta Delta C(T)) method. Methods 25: 402-408, 2001.

19. Dweep H and Gretz N: miRWalk2.0: A comprehensive atlas of microRNA-target interactions. Nat Methods 12: 697, 2015.

20. Jiao X, Sherman BT, Huang da W, Stephens R, Baseler MW, Lane HC and Lempicki RA: DAVID-WS: A stateful web service to facilitate gene/protein list analysis. Bioinformatics 28: 1805-1806, 2012

21. Tabas-Madrid D, Nogales-Cadenas R and Pascual-Montano A: GeneCodis3: A non-redundant and modular enrichment analysis tool for functional genomics. Nucleic Acids Res 40 (Web Server issue): W478-W483, 2012.

22. Shannon P, Markiel A, Ozier O, Baliga NS, Wang JT, Ramage D, Amin N, Schwikowski B and Ideker T: Cytoscape: A software environment for integrated models of biomolecular interaction networks. Genome Res 13: 2498-2504, 2003.

23. Li H, Zhao H, Xue F, Zhang X, Zhang D, Ge J, Yang Y, Xuan M, $\mathrm{Fu} R$ and Yang R: Reduced expression of miR409-3p in primary immune thrombocytopenia. Br J Haematol 161: 128-135, 2013.

24. Guo Y, Qu W, Wang YH, Liu H, Li LJ, Wang HQ, Fu R, Liu H, $\mathrm{Wu} \mathrm{YH}, \mathrm{Guan} \mathrm{J}$, et al: The role of miR-155 in pathogenesis of immune thrombocytopenia. Zhonghua Yi Xue Za Zhi 96: 1103-1107, 2016 (In Chinese)

25. Bay A, Coskun E, Oztuzcu S, Ergun S, Yilmaz F and Aktekin E: Plasma microRNA profiling of pediatric patients with immune thrombocytopenic purpura. Blood Coagul Fibrinolysis 25 379-383, 2014

26. Kottke-Marchant K: Importance of platelets and platelet response in acute coronary syndromes. Cleve Clin J Med 76 (Suppl 1): S2-S7, 2009.
27. Girardot M, Pecquet C, Boukour S, Knoops L, Ferrant A, Vainchenker W, Giraudier S and Constantinescu SN: miR-28 is a thrombopoietin receptor targeting microRNA detected in a fraction of myeloproliferative neoplasm patient platelets. Blood 116: 437-445, 2010.

28. Nagalla S, Shaw C, Kong X, Kondkar AA, Edelstein LC, Ma L, Chen J, McKnight GS, López JA, Yang L, et al: Platelet microRNA-mRNA coexpression profiles correlate with platelet reactivity. Blood 117: 5189-5197, 2011.

29. Yu S, Huang H, Deng G, Xie Z, Ye Y, Guo R, Cai X, Hong J, Qian D, Zhou X, et al: miR-326 targets antiapoptotic Bcl-xL and mediates apoptosis in human platelets. PLoS One 10: e0122784, 2015.

30. Weyrich AS, Schwertz H, Kraiss LW and Zimmerman GA: Protein synthesis by platelets: Historical and new perspectives. J Thromb Haemost 7: 241-246, 2009.

31. McRedmond JP, Park SD, Reilly DF, Coppinger JA, Maguire PB, Shields DC and Fitzgerald DJ: Integration of proteomics and genomics in platelets: A profile of platelet proteins and platelet-specific genes. Mol Cell Proteomics 3: 133-144, 2004.

32. Gnatenko DV, Perrotta PL and Bahou WF: Proteomic approaches to dissect platelet function: Half the story. Blood 108: 3983-3991, 2006.

33. Colombo G, Gertow K, Marenzi G, Brambilla M, De Metrio M, Tremoli E and Camera M: Gene expression profiling reveals multiple differences in platelets from patients with stable angina or non-ST elevation acute coronary syndrome. Thromb Res 128: 161-168, 2011

34. Rowley JW and Weyrich AS: Coordinate expression of transcripts and proteins in platelets. Blood 121: 5255-5256, 2013.

35. Harrison P and Goodall AH: 'Message in the platelet'-more than just vestigial mRNA. Platelets 19: 395-404, 2008.

36. Zhang HW, Zhou P, Wang KZ, Liu JB, Huang YS, Tu YT, Deng ZH, Zhu XD and Hang YL: Platelet proteomics in diagnostic differentiation of primary immune thrombocytopenia using SELDI-TOF-MS. Clin Chim Acta 455: 75-79, 2016.

37. Qiao J, Liu Y, Li D, Wu Y, Li X, Yao Y, Niu M, Fu C, Li H, Ma P, et al: Imbalanced expression of Bcl-xL and Bax in platelets treated with plasma from immune thrombocytopenia. Immunol Res 64: 604-609, 2016.

38. Mitchell WB, Pinheiro MP, Boulad N, Kaplan D, Edison MN, Psaila B, Karpoff M, White MJ, Josefsson EC, Kile BT and Bussel JB: Effect of thrombopoietin receptor agonists on the apoptotic profile of platelets in patients with chronic immune thrombocytopenia. Am J Hematol 89: E228-E234, 2014

39. Winkler J, Kroiss S, Rand ML, Azzouzi I, Annie Bang KW, Speer O and Schmugge M: Platelet apoptosis in paediatric immune thrombocytopenia is ameliorated by intravenous immunoglobulin. Br J Haematol 156: 508-515, 2012.

40. Osman A and Fälker K: Characterization of human platelet microRNA by quantitative PCR coupled with an annotation network for predicted target genes. Platelets 22: 433-441, 2011.

41. Zhang W, Wan M, Ma L, Liu X and He J: Protective effects of ADAM8 against cisplatin-mediated apoptosis in non-small-cell lung cancer. Cell Biol Int 37: 47-53, 2013.

42. Kroll H, Sun QH and Santoso S: Platelet endothelial cell adhesion molecule-1 (PECAM-1) is a target glycoprotein in drug-induced thrombocytopenia. Blood 96: 1409-1414, 2000.

43. Ulger Z, Aksu S, Aksoy DY, Koksal D, Haznedaroglu IC and Kirazli S: The adhesion molecules of L-selectin and ICAM-1 in thrombocytosis and thrombocytopenia. Platelets 21: 49-52, 2010. 